\title{
A novel method for fabricating hybrid biobased nanocomposites film with stable fluorescence containing CdTe quantum dots and montmorillonite-chitosan nanosheets
}

\author{
Yawen Guo ${ }^{\mathrm{a}, \mathrm{b}, 1}$, Xuesong Ge ${ }^{\mathrm{a}, 1}$, Jing Guan ${ }^{\mathrm{a}}$, Lin $\mathrm{Wu}^{\mathrm{c}}$, Fuhua Zhao ${ }^{\mathrm{a}}$, Hui $\mathrm{Li}^{\mathrm{a}}$, Xindong Mu ${ }^{\mathrm{a}}$, \\ Yijun Jiang ${ }^{\mathrm{a}, *}$, Aibing Chen ${ }^{\mathrm{b}, *}$ \\ a Key Laboratory of Bio-based Materials, Qingdao Institute of Bioenergy and Bioprocess Technology, Chinese Academy of Sciences, Qingdao 266101, China \\ ${ }^{\mathrm{b}}$ College of Chemistry and Pharmaceutical Engineering, Hebei University of Science and Technology, Shijiazhuang 050018, China \\ c Qingdao Technical College, Qingdao, Shandong Province 266000, China
}

\section{A R T I C L E I N F O}

\section{Article history:}

Received 6 November 2015

Received in revised form 3 March 2016

Accepted 5 March 2016

Available online 10 March 2016

\section{Keywords:}

Biobased materials

CdTe quantum dots

Montmorillonite-chitosan nanosheets

Fluorescence

Nanocomposites

\begin{abstract}
A B S T R A C T
A method was presented for fabricating the fluorescent nanocomposites containing CdTe quantum dots (QDs) and montmorillonite (MMT)-chitosan (CS). MMT-CS/CdTe QDs nanocomposites were prepared via a simple, versatile and robust approach combination of covalent and electrostatic assembly methods (Scheme 1). The negatively charged MMT was initially modified with positively charged CS through electrostatic assembly, followed by incorporation of CdTe-QDs into the MMT-CS nanosheets by covalent connections between the amino groups of CS and the carboxylic acid groups of thioglycollic acid (TGA). The X-ray diffraction (XRD), High resolution transmission electron microscopy (HRTEM), scanning electron microscopy (SEM) and the FTIR were used to prove the QDs have intercalated into the MMT-CS matrix. The fluorescence emission spectra showed that the MMT-CS/CdTe QDs nanocomposites had the best fluorescence intensity compared with the bare CdTe QDs and CS-QDs.
\end{abstract}

(c) 2016 Elsevier Ltd. All rights reserved.

\section{Introduction}

Chitosan is the principle de-acetylated derivative of chitin which is the second most abundant natural biopolymer after cellulose. Chitosan is a cationic copolymer composed of randomly distributed $\mathrm{N}$-acetyl glucosamine and D-glucosamine, which exhibits some advantages such as nontoxicity, biodegradability and biocompatibility (Pan, Wu, Bao, \& Li, 2011). Chitosan can interact with bio-macromolecules without degrading them. Moreover, chitosan is a positively charged, naturally occurring polysaccharide (Samal et al., 2012). For this reason, chitosan can interact strongly with negatively charged entities, including many lipids and proteins. Owing to the unique characters mentioned above, chitosan has been extensively investigated for several decades for drug delivery, tissue engineering, water engineering, and so on (Chen, Chen, Bai, \& Li, 2013; Croisier \& Jérôme, 2013; Zhang, Chan, \& Leong, 2013).

\footnotetext{
* Corresponding authors.

E-mail addresses: jiangyj@qibebt.ac.cn, muxd@qibebt.ac.cn (Y. Jiang), chen_ab@163.com (A. Chen).

1 These authors contributed equally to this work.
}

On the other hand, the development of bio-hybrid nanostructured materials has received more attention in recent years (El Kadib \& Bousmina, 2012; Ruiz-Hitzky et al., 2013). Inorganic nanostructured composites have unique mechanical, thermal, electronic or photic performance compared with the organic ones. A current trend in materials science involves the development of materials with special structure and character based on the combination of natural polymers with inorganic composites. The inorganic filler materials including clay minerals (Darder et al., 2006), layered double hydroxide (Wei et al., 2012), metal nanoparticles (Nandanan, Jana, \& Ying, 2008), metal oxide nanoparticles (Petkova et al., 2014), carbon nanotube (Pan, Bao, \& Li, 2011), graphene (Fan et al., 2010) and graphene oxide (Lin et al., 2012) have been employed to fabricate the hybrid nanostructured materials with natural polymers. What's more, semiconductor quantum dots (QDs) also have attracted considerable attention due to their unique properties including size-dependent optical properties, high photo-stability and narrow emission spectra compared with the conventional organic dyes (Kim, Woo, Jeong, \& Han, 2011; Liang et al., 2013; Liang et al., 2014; Liu, Shi, Yu, Yang, \& Zuo, 2010). QDs have been widely used in the fields of fluorescent materials(Zhang \& Chen, 2014), optical bioimaging (Li \& Zhu, 2013; Luo et al., 2013; Wu \& Yan, 2013), photovoltaic devices (Chuang, Brown, Bulović, \& Bawendi, 
2014; Kamat, 2013) and light-emitting diodes (Dai et al., 2014). Among various QDs materials, CdTe QDs possess unique fluorescence property. However CdTe QDs displayed a strong tendency towards oxidization and agglomeration due to its nanosize effects, especially in polymers (Xu et al., 2013). So, it is desirable to improve the compatibility and stability of these nanocomposites in biopolymers.

In this work, a novel method was employed to fabricate stable MMT-CS/CdTe hybrid nanocomposites. In this process, chitosan was first intercalated into montmorillonite to get MMT-CS hybrid composites, and then the CdTe QDs were in situ intercalated into MMT-CS to obtain MMT-CS/CdTe hybrid nanocomposites. The assynthesized composites can show excellent fluorescence property in aqueous solution. Moreover, a film containing MMT-CS/CdTe with artificial nacre-like structure can also be produced by simple filtration. Importantly, the multilayer hybrid films of MMT-CS/CdTe obtained by a simple filtration method also present strong and stable fluorescence, which have potential use in optical or electronic applications.

\section{Experiment}

\subsection{Materials}

Sodium montmorillonite (Na-MMT, G105-PGW) nanoclays (particle size $=16-22 \mu \mathrm{m}$; aspect ratio $=200-400$ ) were purchased from NANOCOR. Chitosan ( $\geq 95 \%$ deacetylate; viscosity $\left._{2 \%}=100-200 \mathrm{mPa} . \mathrm{s}\right)$, thioglycolic acid (TGA, AR, 90\%) and $\mathrm{CdCl}_{2} \cdot 2.5 \mathrm{H}_{2} \mathrm{O}$ (99.95\%) were purchased from Aladdin. $\mathrm{NaBH}_{4}$ (96\%), tellurium (Te) power, acetic acid $(\mathrm{AR}, \geq 99.5 \%)$ and $\mathrm{NaOH}$ (AR, $\geq 99.5 \%$ ) were purchased from Sinopharm Chemical Reagent Co. Ltd. The water used in this work was deionized water.

\subsection{Preparation of MMT-chitosan nanosheets}

MMT-chitosan nanosheets were prepared as follows (Yao, Tan, Fang, \& Yu, 2010). $0.5 \mathrm{~g}$ MMT was dispersed in $100 \mathrm{~mL}$ of deionized water under vigorous stirring for two days and then the solution was stayed for $24 \mathrm{~h}$ and took the liquid supernatant for subsequent use. Solid content of the supernatant was $0.42 \mathrm{wt} \%$ and the $\mathrm{pH}$ was 10.14. $100 \mathrm{~mL}$ of $2 \mathrm{wt} \%$ chitosan ( $2 \mathrm{wt} \%$ of chitosan were prepared by dissolving chitosan in an aqueous solution of $2 \mathrm{wt} \%$ acetic acid for $24 \mathrm{~h}$ before use) were added into $100 \mathrm{~mL}$ liquid supernatant of the MMT solution and stirred for $6 \mathrm{~h}$ to form MMT-CS mixed solution. The mixture was centrifuged and washed with deionized water to remove the unabsorbed chitosan. Then we dissolved the MMT-CS into $100 \mathrm{~mL}$ deionized water for further use (solid content $=1.02 \%$, $\mathrm{pH}$ 4.02).

\subsection{CdTe QDs fabrication on the structure of MMT-CS nanosheets}

$100 \mathrm{~mL}$ re-dispersion MMT-CS solution and $80 \mathrm{mg} \mathrm{CdCl} 2 \cdot 2.5 \mathrm{H}_{2} \mathrm{O}$ were added into a three-neck bottle. After stirring for $3 \mathrm{~h}$ under $\mathrm{N}_{2}$ atmosphere at room temperature, $72 \mu \mathrm{L}$ TGA was added into the mixture and the $\mathrm{pH}$ of the mixture was adjusted to 9 using $1 \mathrm{~mol} / \mathrm{L}$ $\mathrm{NaOH}$ (Li et al., 2013). After that, a freshly prepared NaHTe (Cao, $2012)$ solution $(1.085 \mu \mathrm{L}, 0.2 \mathrm{~mol} / \mathrm{L})$ was added into above mixed solution. Several reactions were carried out at $135^{\circ} \mathrm{C}$ for different reflux time ( $1 \mathrm{~h}, 2 \mathrm{~h}, 3 \mathrm{~h}, 4 \mathrm{~h})$ to synthesize negatively charged CdTe QDs solutions. The method to synthesis CdTe QDs aqueous solutions without MMT-CS was almost the same with that of MMTCS/QDs. The only difference was that we used $100 \mathrm{~mL}$ deionized water instead of $100 \mathrm{~mL}$ re-dispersion MMT-CS solution.

\subsection{Fabrication of multilayer films of MMT-CS/QDs}

The multilayer films of MMT-CS/QDs were fabricated by a onestep layer-by-layer (LBL) (Han et al., 2011; Podsiadlo et al., 2007; Yao, Fang, Tan, Wu, \& Yu, 2010) assembly method. A $15 \mathrm{~mL}$ aliquot of the MTM-CS/CdTe QDs nanosheets suspension was vacuum filtrated by a cellulose acetate filter membrane $(0.22 \mu \mathrm{m}$, Jinteng $)$ set on a sand core ( $4 \#, \phi=50 \mathrm{~mm}$ ) funnel. The wet hybrid film was rinsed gently with deionized water and dried at $40^{\circ} \mathrm{C}$ by nitrogen flow. Consequently, the film with average thickness of $0.04 \mathrm{~mm}$ can be easily peeled off from the filter membrane.

\section{Characterization}

\section{1. $X$-ray diffraction $(X R D)$}

The X-ray powder diffraction (XRD) patterns were recorded on a Bruker D8 Advance X-ray diffraction spectrometer using $\mathrm{Cu}-\mathrm{K} \alpha$ radiation at $80 \mathrm{kV}$ and $40 \mathrm{~mA}$. The scanning rate was $4 \% \mathrm{~min}$ and the scanning scope of $2 \theta$ was $5-50^{\circ}$ at room temperature.

\subsection{Fourier-transform infrared (FTIR)}

Fourier-transform infrared (FTIR) spectra of the samples were recorded on a Nicolet 6700 FTIR spectrometer (Thermo Fisher) in the frequency range of $4000-400 \mathrm{~cm}^{-1}$. The specimens were prepared by the $\mathrm{KBr}$ disk method.

\subsection{Transmission electron microscopy (TEM)}

Transmission electron microscopy (TEM) was performed using an $\mathrm{H}-7650$ Hitachi transmission electron microscope operating at $160 \mathrm{kV}$. High resolution transmission electron microscopy (HRTEM) and selected area electron diffraction (SAED) patterns were carried out using a FEI Tecnai G2 F30 S-Twin transmission electron microscope operating at $200 \mathrm{kV}$. All the samples were diluted 20 times in water and then dried on the carbon-coated copper grid for the TEM measurements.

\subsection{Scanning electron microscope (SEM)}

The morphology of films was investigated by using a scanning electron microscope (SEM Hitachi S-4800) equipped with an energy dispersive spectroscopy (EDS) attachment (EMAX), and the accelerating voltage applied was $20 \mathrm{kV}$. The films were cooled in liquid nitrogen and then broken, and the fracture surfaces were vacuum coated with gold for SEM.

\subsection{Ultraviolet absorption and fluorescence emission}

UV-vis absorption spectra were recorded on a Hitachi U-4100 spectrometer. Fluorescence spectra were recorded using a Hitachi F-4600 spectrometer at room temperature with excitation wavelength at $360 \mathrm{~nm}$. All the solutions were diluted 50 times and all the films were cut into strip-type and fixed on a special fixture before UV-vis and fluorescence measurements.

\subsection{Thermo gravimetric analysis (TGA)}

Thermal properties of films were measured with a thermo gravimetric analyzer (Shimadzu, model DTG-60, Kyoto, Japan). The sample was heated from $25^{\circ} \mathrm{C}$ to $600^{\circ} \mathrm{C}$ with a heating rate of $10^{\circ} \mathrm{C} / \mathrm{min}$ in a nitrogen flow $(30 \mathrm{~mL} / \mathrm{min})$ to avoid thermo-oxidative degradation. 


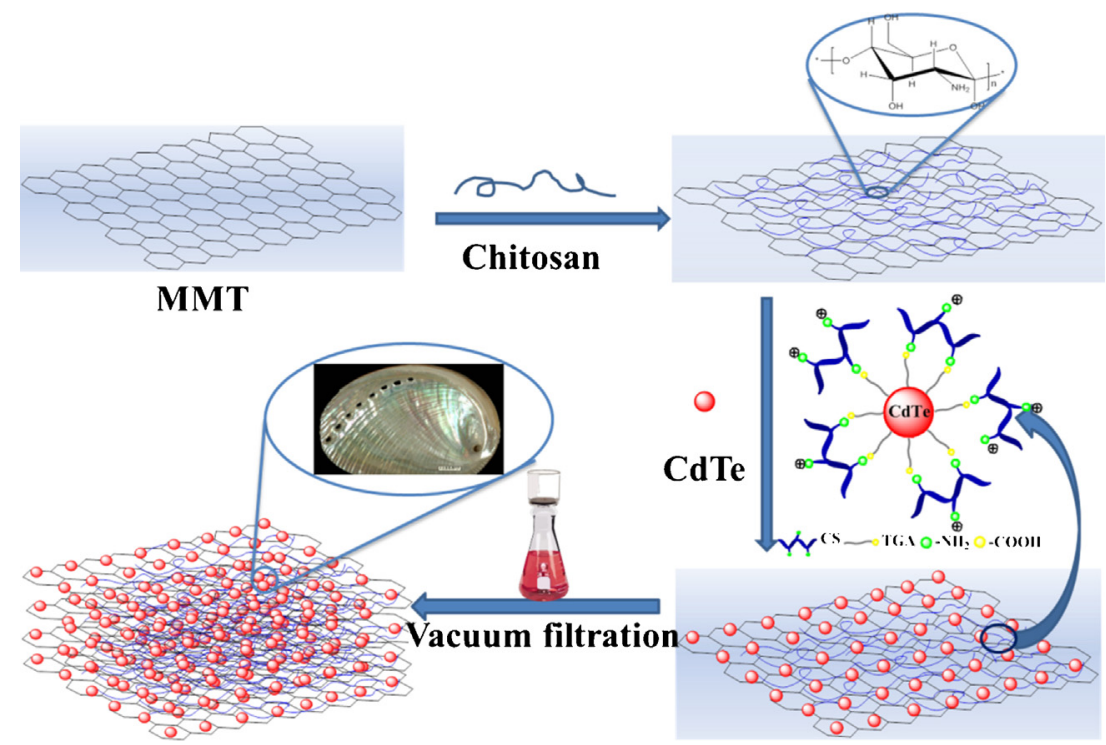

Scheme 1. Graphic abstract for the LBL fabrication of multilayer luminous films based on MMT-CS/CdTe QDs nanoplatelets.

\section{Results and discussion}

MMT-CS/CdTe QDs hybrid nanocomposites were prepared via a simple, versatile and robust approach combination of covalent and electrostatic assembly methods. The fabrication procedure of the nanocomposites is demonstrated in Scheme 1. The negatively charged MMT were initially modified with positively charged CS through electrostatic assembly, followed by incorporation of CdTeQDs into the MMT-CS nanosheets by covalent connections between the amino groups of CS and the carboxylic acid groups of thioglycollic acid (TGA).

\subsection{Preparation of MMT-chitosan nanosheets}

MMT-chitosan nanosheets were prepared via the delamination of commercially available natural MMT particles (particle size $=16-22 \mu \mathrm{m}$; aspect ratio $=200-400$ ) in deionized water and mixed with the positively charged CS through electrostatic assembly. The procedure were examined by XRD, TEM, IR and TG to prove the exfoliated MMT was a monolayer and combined with CS.

The changes in the packing state of the MMT lamellae in the MMT-CS and MMT-CS/QDs were effectively detected by the XRD patterns displayed in Fig. 1A. The interlayer distance $d(001)$ of MMT was $1.25 \mathrm{~nm}$ according to the peak at a $2 \theta$ value of $7.06^{\circ}$ (001 peak) using Bragg's equation. However, this peak shifted to $6.27^{\circ}$ and $5.82^{\circ}$ when MMT were added to the CS and modified by QDs, respectively. The interlayer distance of the MMT-CS and MMTCS/QDs changed to $1.41 \mathrm{~nm}$ and $1.52 \mathrm{~nm}$, indicating that the MMT lamellae were intercalated by CS chains and QDs. The intercalation of MMT was attributed to the electrostatic interaction between CS and MMT. Moreover, the incorporation of CS or CS/QDs flattened the peaks of MMT indicating the reduced crystallinity of the MMT. This might be ascribed to the interactions between MMT and CS chains, which could hinder the ordered packing of the MMT lamellae. The same result was proved by the TEM of the exfoliated MMT and MMT-CS/QDs with reflux time of $2 \mathrm{~h}$ shown in Fig. 1C. The MMT lamellae were coated by CS chains compared with the exfoliated MMT.

The FTIR spectra in the $4000-400 \mathrm{~cm}^{-1}$ wavenumber range of the MMT, CS, MMT-CS and MMT-CS/QDs nanocomposites are shown in Fig. 1B. All of them show strong characteristic bands of MMT for silicate $\left(v_{(\mathrm{OH})}\right.$ of $\mathrm{Al}, \mathrm{Mg}(\mathrm{OH}) \sim 3632 \mathrm{~cm}^{-1} ; v_{(\mathrm{OH})}$ of $\mathrm{H}_{2} \mathrm{O} \sim 3440 \mathrm{~cm}^{-1} ; \delta_{\mathrm{HOH}} \sim 1650 \mathrm{~cm}^{-1}$; and $\nu_{(\mathrm{SiO})}$ of $\mathrm{Si}-\mathrm{O}-\mathrm{Si}$ $\sim 1040 \mathrm{~cm}^{-1}$ ). The bands attributed to the intercalated chitosan are also observed in the spectra of MMT-CS and MMT-CS/QDs. The frequency of vibrational bands at $1645 \mathrm{~cm}^{-1}$ in the pure chitosan, which corresponds to the deformation vibration of the protonated amine group $\left(\delta_{\mathrm{NH} 3}\right)$ is shifted toward lower frequency values $\left(1556 \mathrm{~cm}^{-1}\right.$ in MMT-CS and $1567 \mathrm{~cm}^{-1}$ in MMT-CS/QDs) after combing with CS. This result can be related to the electrostatic interaction between such groups and the negatively charged sites in the MMT lamellae, which proved CS was intercalated into the MMT layers. The vibrational bands $v_{(\mathrm{CO})}$ of $\mathrm{C}-$ $\mathrm{O}-\mathrm{C} \sim 1075 \mathrm{~cm}^{-1}$ (characteristic of the pyranose ring) and $\nu_{(\mathrm{OH})}$ $\sim 3435 \mathrm{~cm}^{-1}$ in the pure chitosan overlap with the bands of the silicate.

\subsection{Modification of MMT-CS nanosheets by CdTe QDS}

The CdTe-QDs inserted into the MMT-CS nanosheets through covalent connections between the amino groups of CS and the carboxylic acid groups of thioglycollic acid (TGA). Fig. 2 is the HRTEM of the MMT-CS nanosheets modified by CdTe QDs with different reflux time. As depicted in the HRTEM images in Fig. 2(A-D), the newly formed CdTe QDs dispersed very well on the surface of MMT$\mathrm{CS}$. However, the CdTe QDs gathered when the reflux time was $4 \mathrm{~h}$. The insets in Fig. 2A-D are the mean size of the CdTe QDs calculated from the HRTEM images. The mean size of the CdTe QDs increased with prolonging the reflux time. The mean size of the CdTe QDs with reflux time of $1 \mathrm{~h}$ was found to be $1.23 \mathrm{~nm}$. The particle size increased to $3.21 \mathrm{~nm}$ when the reflux time was $4 \mathrm{~h}$ and the CdTe QDs began to form clusters. Mean size and fluorescence properties of the CdTe QDs are summarized in Table 1. The SAED pattern of the CdTe QDs with reflux time of $2 \mathrm{~h}$ shows sharp diffraction rings, indicating that the CdTe nanoparticles are well crystalline.

The modification procedure with different refluxing time was also monitored by UV-vis adsorption and fluorescence emission spectra. As shown in Fig. S1, the UV and fluorescence spectra of the MMT-CS/QDs exhibit a red-shift with prolonging of the refluxing time which accelerates the Ostwald's ripening of the nanocrystals. This can be explained by the size increase of the CdTe QDs shown in the TEM images (Fig. 2). CdTe QDs are electronic structure intermediates between bands and bonds which result in a direct correlation between the size and band gap energy (emitted 

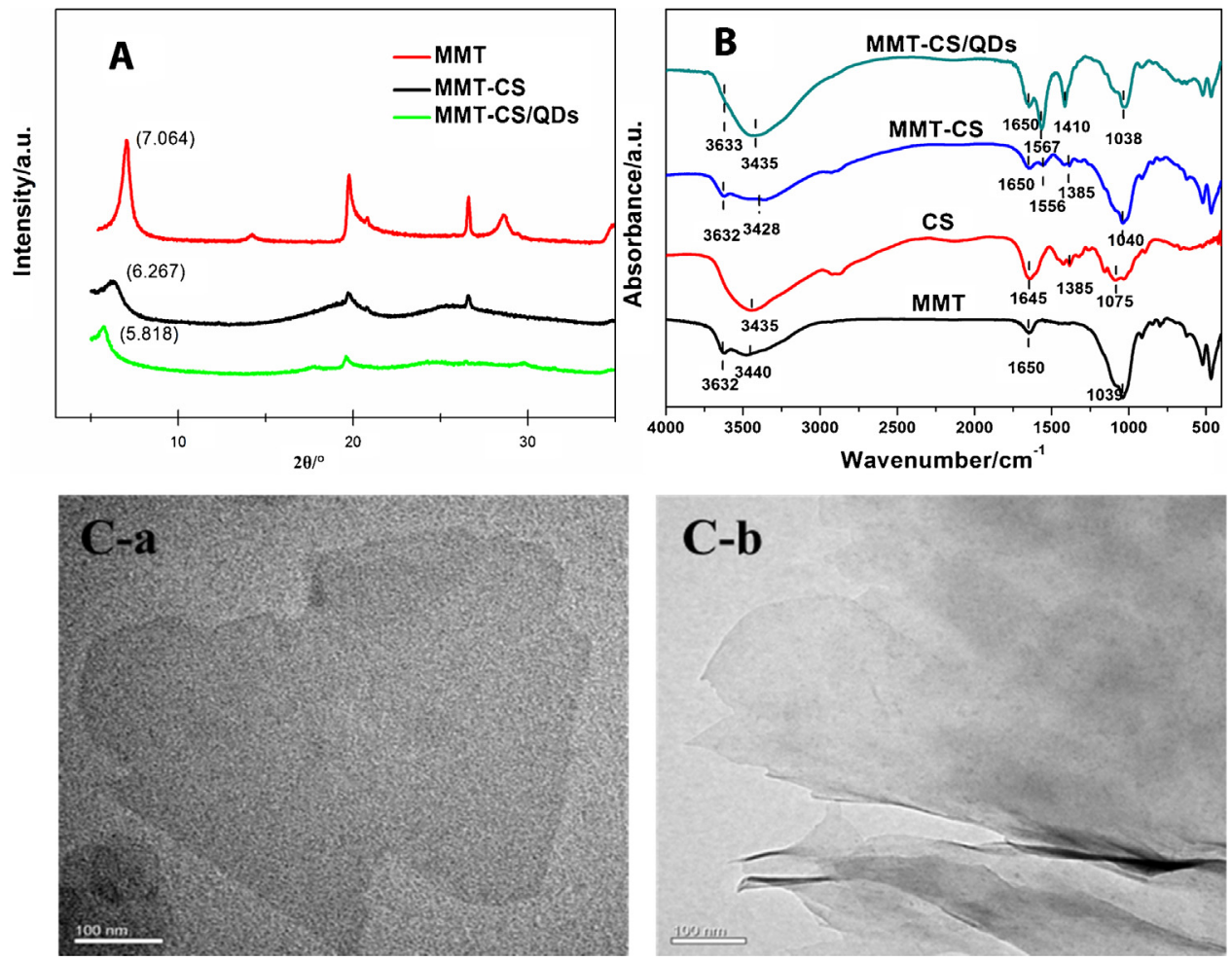

Fig. 1. (A) XRD patterns of the exfoliated MMT, MMT-CS and MMT-CS/QDs; (B) FTIR spectra of the exfoliated MMT, CS, MMT-CS and MMT-CS/QDs; (C) TEM image of the exfoliated MMT nanosheets; (a) and the MMT-CS/QDs with reflux time of $2 \mathrm{~h}(\mathrm{~b})$.

Table 1

Mean size and fluorescence properties of the CdTe QDs.

\begin{tabular}{|c|c|c|c|c|}
\hline \multirow[t]{2}{*}{ Samples } & \multirow[t]{2}{*}{ Reflux time $(\mathrm{h})$} & \multirow[t]{2}{*}{ Mean size of $\mathrm{QDs}^{\mathrm{a}}(\mathrm{nm})$} & \multicolumn{2}{|c|}{ Maximum emission wavelength ( $\mathrm{nm}$ ) } \\
\hline & & & solution & films \\
\hline MMT-CS & 0 & - & - & - \\
\hline MMT-CS/CdTe QDs-1 & 1 & $1.23 \pm 0.23$ & 551 & 541 \\
\hline MMT-CS/CdTe QDs-2 & 2 & $2.05 \pm 0.46$ & 560 & 544 \\
\hline MMT-CS/CdTe QDs-3 & 3 & $2.44 \pm 0.22$ & 565 & 552 \\
\hline MMT-CS/CdTe QDs-4 & 4 & $3.21 \pm 0.31$ & 590 & 560 \\
\hline
\end{tabular}

a The mean size of the CdTe QDs was calculated from the HRTEM images by software named SmileView.

wavelength). As the size of the QD increases, the band gap energy decreases which result in the re-shift of the UV and fluorescence spectra (Baskoutas \& Terzis, 2006; Poznyak et al., 2005). Besides, the MMT-CS/QDs aqueous solution exist strong fluorescence intensity when the reflux time was less than $2 \mathrm{~h}$ as shown in Fig. 3A. However, the fluorescence intensity decreased when the reflux time was $3 \mathrm{~h}$ or more. This can be ascribed to the fact that the CdTe QDs began to form clusters and made fluorescence quenching with the reflux time increasing. The photographs of the MMT-CS/QDs under UV irradiation were also displayed in the inset of Fig. 3A. Visually, a color change from yellow-green to yellow and red along with a decrease in brightness can be clearly observed as the increasing of reflux time (Fig. 3A).

We also compared the fluorescence intensity of QDs, QDs-CS, QDs-MMT and MMT-CS/QDs with the same reflux time at $2 \mathrm{~h}$ and the same concentration of QDs (Fig. 3B). As we can see, MMTCS/QDs aqueous solution shows the best fluorescence intensity (35\% higher than the pure CdTe QDs). This might be ascribed to the connections between CS chains and CdTe QDs. The CS chains limit the movement of CdTe QDs and reduce the aggregation among them. In addition, the storage stability test on the MMT-CS/QDs aqueous solution shows that $\sim 82.5 \%$ of its original fluorescence intensity remained after four weeks while the simple QDs only remained about $60.3 \%$ (Fig. S2 in Supporting information). The results above indicate that the MMT-CS/QDs aqueous solution possesses strong fluorescence intensity with its wavelength controllable and good storage stability.

\subsection{Fabrication of multilayer films of MMT-CS/QDs}

The multilayer films of MMT-CS/QDs were fabricated using a one-step LBL method. The cross section and surface microstructures of MMT-CS/QDs hybrid films were characterized by scanning electron microscopy (SEM) to confirm the formation of welldefined nacre-like layered structure hybrid coatings. As shown in Fig. 4A, MMT-CS/QDs hybrid nanosheets were arranged on the substrate forming nacre-like lamellar microstructure. The SEM image of the surface (Fig. 4B) indicates that hybrid nanosheets are almost flatly stacked.

In order to know the relative content of the elements in the membrane, EDX spectra was obtained as shown in Fig. 4C. From the image of the EDX spectra, it can be seen the element characteristic peaks of Cd and Te. Thermal stability was investigated from TG curves recorded in the $25-600^{\circ} \mathrm{C}$ range, under air flow conditions (Fig. 4D). The weight loss between $25^{\circ} \mathrm{C}$ and $230^{\circ} \mathrm{C}$ is related to the adsorbed water molecules. The weight loss between $230^{\circ} \mathrm{C}$ and 

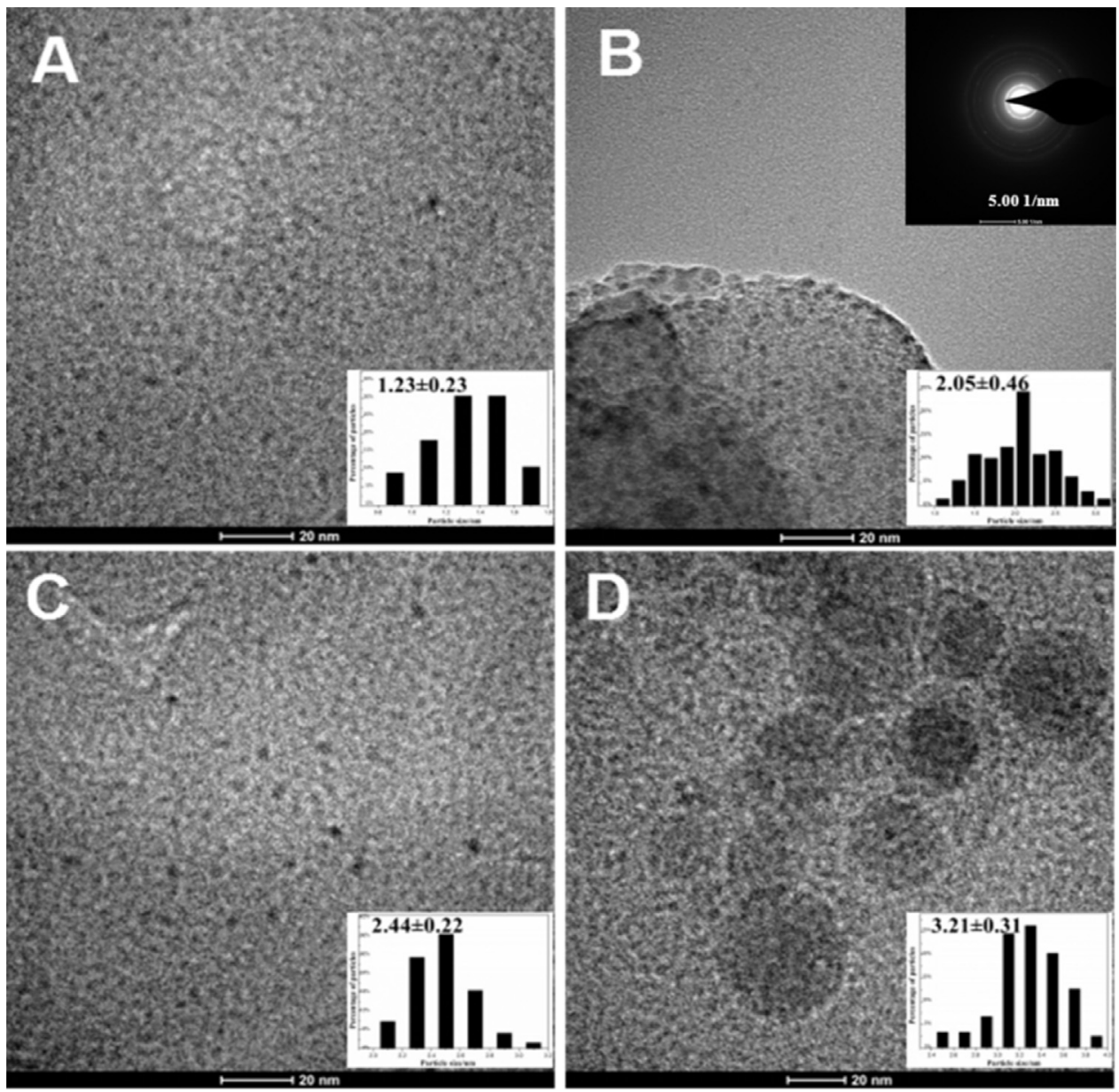

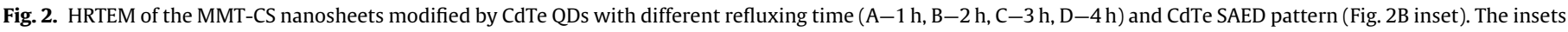
were mean size of the CdTe QDs calculated from HRTEM by software named SmileView.
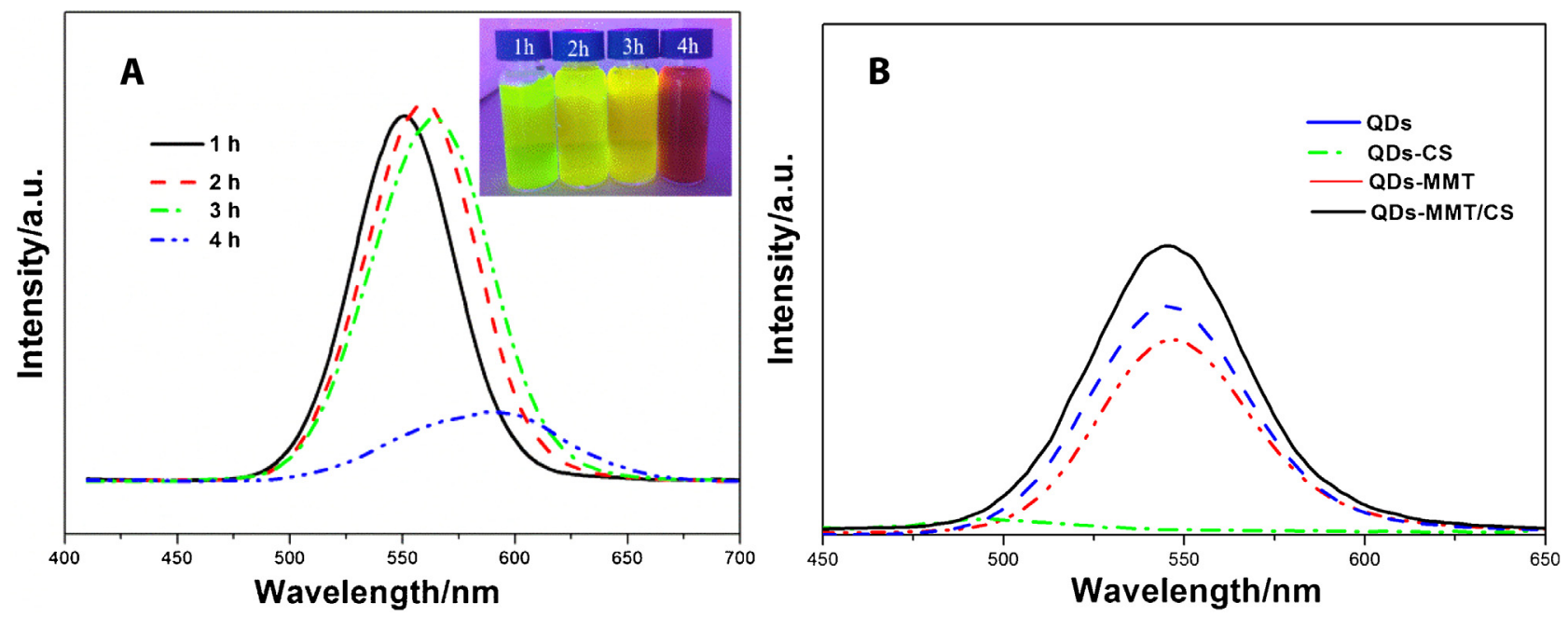

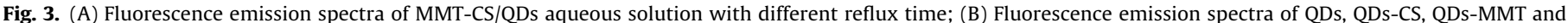
QDs-MMT-CS with reflux time of $2 \mathrm{~h}$.

$600^{\circ} \mathrm{C}$ correspond to the combustion of the intercalated chitosan. As we expected, MMT showed excellent thermal stability, with a residue as high as $95.87 \mathrm{wt} \%$ at $600^{\circ} \mathrm{C}$, while CS displayed a residue of only $37.81 \mathrm{wt} \%$. Based on the great difference of the weight loss between MMT and CS, ranging from $230^{\circ} \mathrm{C}$ to $600^{\circ} \mathrm{C}$, the content of CS that coated onto the MMT lamellae could be estimated and was approximately $44.82 \mathrm{wt} \%$. However, the MMT-CS and MMT-CS/QDs films displayed similar thermal degradation behaviors compared with the pure CS film. Therefore, we conclude that the CS chains in MMT-CS mostly remained coated on the surface of MMT lamellae.

The films of MMT-CS/QDs were fabricated by a one-step LBL assembly method and their fluorescence emission spectra are 

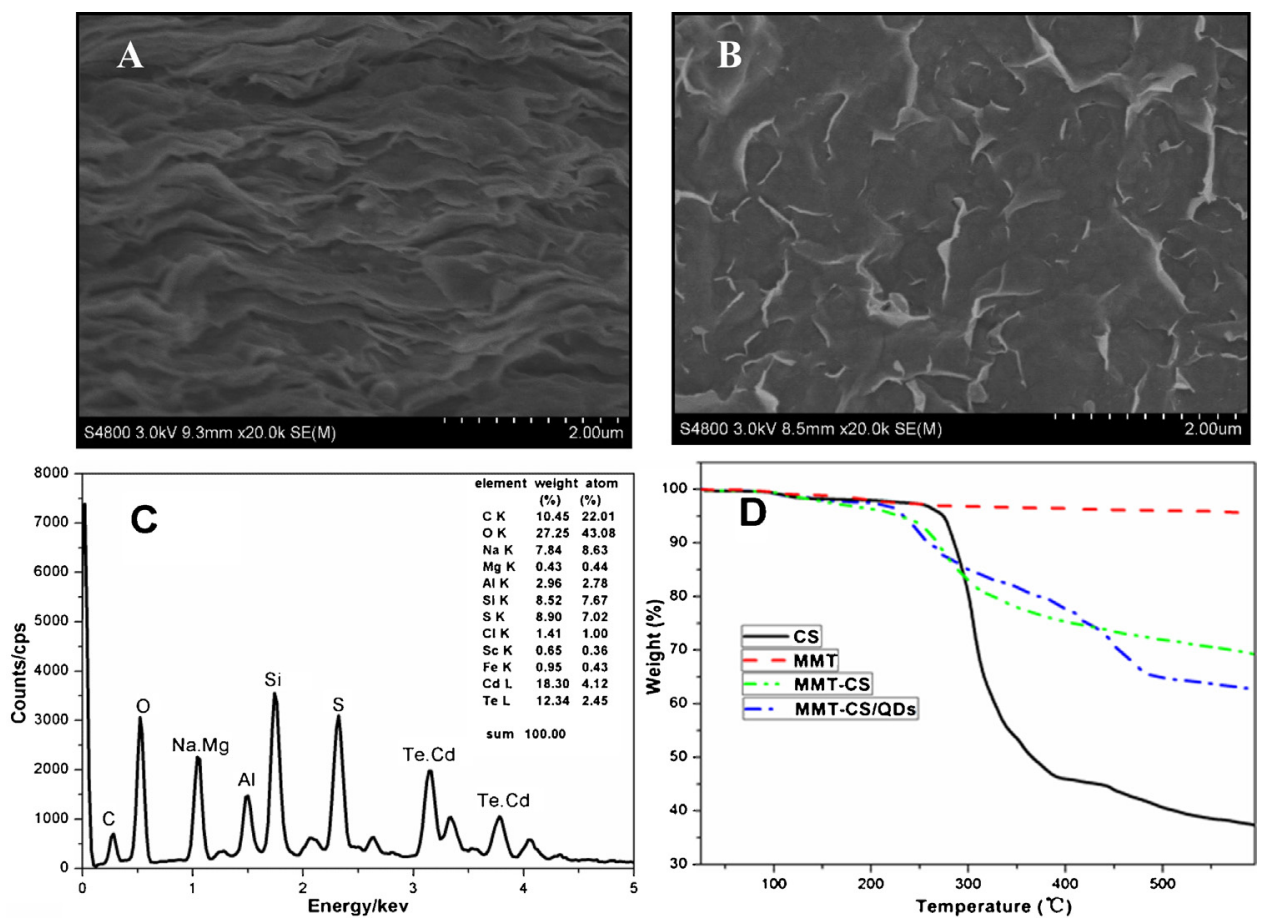

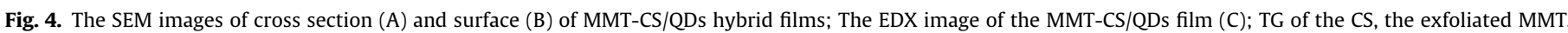
MMT-CS and the MMT-CS/QDs (D).
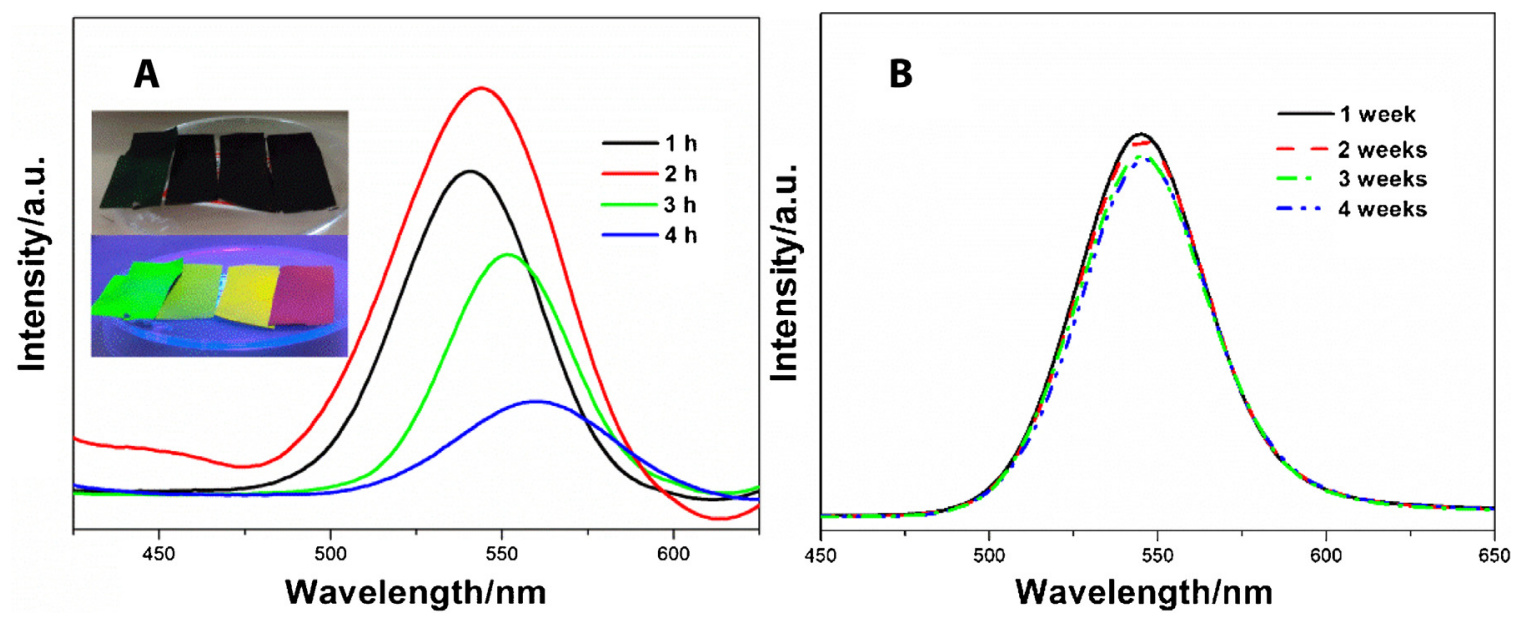

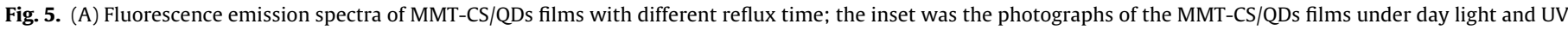
irradiation. (B) Fluorescence emission spectra of the MMT-CS/QDs films with reflux time of $2 \mathrm{~h}$ (stored at ambient temperature) recorded weekly in 4 weeks.

shown in Fig. 5A. The MMT-CS/QDs films show similar fluorescence property with its solution. The spectra exhibit a red-shift with prolonging of the refluxing time. The photographs of the MMTCS/QDs films under day light and UV irradiation were also displayed in the inset of Fig. 5A. Visually, all the films show strong fluorescence intensity and a color change from yellow-green to yellow and red can be clearly observed as the increasing of reflux time. Besides, the MMT-CS/QDs films also possess good storage stability with $\sim 92.3 \%$ of its original fluorescence intensity remained after four weeks (Fig. 5B).

\section{Conclusion}

In summary, we report a feasible route to fabricate an organicinorganic nacre-like hybrid film using covalent and electrostatic assembly methods. This method allows the accurate control of the
QDs' size by refluxing the mixture with different time. Remarkably, the MMT-CS/QDs films presented strong fluorescence property remained after four weeks and good storage stability. The simple preparation and tunable fluorescence property of the MMT-CS/QDs nanocomposites provide a platform for the engineering and assembling of advanced materials for potential use in an exciting range of optical or electronic applications.

\section{Acknowledgements}

This work was supported by K.C. Wong Education Foundation, Youth Innovation Promotion Association of CAS; the Qingdao Institute of Bioenergy and Bioprocess Technology Director Technology Foundation; Shandong Provincial Natural Science Foundation for Distinguished Young Scholar, China (No. JQ201305), China 
and CAS-TWAS President's PhD Fellowship programme of K.S (2013A8017908241).

\section{Appendix A. Supplementary data}

Supplementary data associated with this article can be found, in the online version, at http://dx.doi.org/10.1016/j.carbpol.2016.03. 016.

\section{References}

Baskoutas, S., \& Terzis, A. F. (2006). Size-dependent band gap of colloidal quantum dots. Journal of Applied Physics, 99(1), 013708.

Cao, Y.-C. (2012). Preparation of thermally stable well-dispersed water-soluble CdTe quantum dots in montmorillonite clay host media. Journal of Colloid and Interface Science, 368(1), 139-143.

Chen, Y., Chen, L., Bai, H., \& Li, L. (2013). Graphene oxide-chitosan composite hydrogels as broad-spectrum adsorbents for water purification. Journal of Materials Chemistry A, 1(6), 1992-2001.

Chuang, C.-H. M., Brown, P. R., Bulović, V., \& Bawendi, M. G. (2014). Improved performance and stability in quantum dot solar cells through band alignment engineering. Nature Materials, 13(8), 796-801.

Croisier, F., \& Jérôme, C. (2013). Chitosan-based biomaterials for tissue engineering. European Polymer Journal, 49(4), 780-792.

Dai, X., Zhang, Z., Jin, Y., Niu, Y., Cao, H., Chen, X., Wang, L., Liang, J., \& Peng, X. (2014). Solution-processed, high-performance light-emitting diodes based on quantum dots. Nature, 515(7572), 96-99.

Darder, M., López-Blanco, M., Aranda, P., Aznar, A. J., Bravo, J., \& Ruiz-Hitzky, E (2006). Microfibrous chitosan-sepiolite nanocomposites. Chemistry of Materials, 18(6), 1602-1610.

El Kadib, A., \& Bousmina, M. (2012). Chitosan bio-based organic-inorganic hybrid aerogel microspheres. Chemistry-A European Journal, 18(27), 8264-8277.

Fan, H., Wang, L., Zhao, K., Li, N., Shi, Z., Ge, Z., \& Jin, Z. (2010). Fabrication, mechanical properties, and biocompatibility of graphene-reinforced chitosan composites. Biomacromolecules, 11(9), 2345-2351.

Han, J., Dou, Y., Yan, D., Ma, J., Wei, M., Evans, D. G., \& Duan, X. (2011). Biomimetic design and assembly of organic-inorganic composite films with simultaneously enhanced strength and toughness. Chemical Communications (Cambridge), 47(18), 5274-5276.

Kamat, P. V. (2013). Quantum dot solar cells. The next big thing in photovoltaics. The Journal of Physical Chemistry Letters, 4(6), 908-918.

Kim, K., Woo, J. Y., Jeong, S., \& Han, C. S. (2011). Photoenhancement of a quantum dot nanocomposite via UV annealing and its application to white LEDs. Advanced Materials, 23(7), 911-914.

Li, H., Liu, J., Wang, K., Yu, X., Zuo, S., \& Li, B. (2013). Preparation of fluorescent $\mathrm{CdTe} / \mathrm{montmorillonite} \mathrm{nanocomposites} \mathrm{and} \mathrm{their} \mathrm{incorporation} \mathrm{into}$ polypropylene. Materials Letters, 107, 284-286.

Li, J., \& Zhu, J. (2013). Quantum dots for fluorescent biosensing and bio-imaging applications. Analyst, 138(9), 2506-2515.

Liang, R., Tian, R., Shi, W., Liu, Z., Yan, D., Wei, M., \& Duan, X. (2013). A temperature sensor based on CdTe quantum dots-layered double hydroxide ultrathin films via layer-by-layer assembly. Chemical Communications (Cambridge), 49(10), 969-971.

Liang, R., Yan, D., Tian, R., Yu, X., Shi, W., Li, C., \& Duan, X. (2014). Quantum dots-based flexible films and their application as the phosphor in white light-emitting diodes. Chemistry of Materials, 26(8), 2595-2600.
Lin, X., Shen, X., Zheng, Q., Yousefi, N., Ye, L., Mai, Y.-W., \& Kim, J.-K. (2012). Fabrication of highly-aligned, conductive, and strong graphene papers using ultralarge graphene oxide sheets. ACS Nano, 6(12), 10708-10719.

Liu, J., Shi, Z., Yu, Y., Yang, R., \& Zuo, S. (2010). Water-soluble multicolored fluorescent CdTe quantum dots: synthesis and application for fingerprint developing. Journal of Colloid and Interface Science, 342(2), 278-282.

Luo, P. G., Sahu, S., Yang, S.-T., Sonkar, S. K., Wang, J., Wang, H., \& Sun, Y.-P. (2013). Carbon quantum dots for optical bioimaging. Journal of Materials Chemistry B, 1(16), 2116-2127

Nandanan, E., Jana, N. R., \& Ying, J. Y. (2008). Functionalization of gold nanospheres and nanorods by chitosan oligosaccharide derivatives. Advanced Materials, 20(11), 2068-2073

Pan, Y., Bao, H., \& Li, L. (2011). Noncovalently functionalized multiwalled carbon nanotubes by chitosan-grafted reduced graphene oxide and their synergistic reinforcing effects in chitosan films. ACS Applied Materials E' Interfaces, 3(12), 4819-4830.

Pan, Y., Wu, T., Bao, H., \& Li, L. (2011). Green fabrication of chitosan films reinforced with parallel aligned graphene oxide. Carbohydrate Polymers, 83(4), 1908-1915.

Petkova, P., Francesko, A., Fernandes, M. M., Mendoza, E., Perelshtein, I., Gedanken, A., \& Tzanov, T. (2014). Sonochemical coating of textiles with hybrid $\mathrm{ZnO} /$ chitosan antimicrobial nanoparticles. ACS Applied Materials E Interfaces, 6(2), 1164-1172.

Podsiadlo, P., Kaushik, A. K., Arruda, E. M., Waas, A. M., Shim, B. S., Xu, J., \& Ramamoorthy, A. (2007). Ultrastrong and stiff layered polymer nanocomposites. Science, 318(5847), 80-83.

Poznyak, S. K., Osipovich, N. P., Shavel, A., Shavel, D. V., Gao, M., Eychmu1ller, A., \& Gaponik, N. (2005). Size-dependent electrochemical behavior of thiol-capped CdTe nanocrystals in aqueous solution. The Journal of Physical Chemistry B, 109(3), 1094-1100.

Ruiz-Hitzky, E., Darder, M., Fernandes, F. M., Wicklein, B., Alcântara, A. C., \& Aranda P. (2013). Fibrous clays based bionanocomposites. Progress in Polymer Science, 38(10), 1392-1414

Samal, S. K., Dash, M., Van Vlierberghe, S., Kaplan, D. L., Chiellini, E., Van Blitterswijk, C., \& Dubruel, P. (2012). Cationic polymers and their therapeutic potential. Chemical Society Reviews, 41(21), 7147-7194.

Wei, P.-R., Cheng, S.-H., Liao, W.-N., Kao, K.-C., Weng, C.-F., \& Lee, C.-H. (2012). Synthesis of chitosan-coated near-infrared layered double hydroxide nanoparticles for in vivo optical imaging. Journal of Materials Chemistry, 22(12), 5503-5513.

Wu, P., \& Yan, X.-P. (2013). Doped quantum dots for chemo/biosensing and bioimaging. Chemical Society Reviews, 42(12), 5489-5521.

Xu, S., Lu, H., Li, J., Song, X., Wang, A., Chen, L., \& Han, S. (2013). Dummy molecularly imprinted polymers-capped CdTe quantum dots for the fluorescent sensing of 2, 4, 6-trinitrotoluene. ACS Applied Materials \& Interfaces, 5(16), 8146-8154.

Yao, H. B., Fang, H. Y., Tan, Z. H., Wu, L. H., \& Yu, S. H. (2010). Biologically inspired, strong, transparent, and functional layered organic-inorganic hybrid films. Angewandte Chemie International Edition in English, 122(12), 2186-2191.

Yao, H. B., Tan, Z. H., Fang, H. Y., \& Yu, S. H. (2010). Artificial nacre-like bionanocomposite films from the self-assembly of chitosan-montmorillonite hybrid building blocks. Angewandte Chemie International Edition, 49(52), 10127-10131.

Zhang, R., \& Chen, W. (2014). Nitrogen-doped carbon quantum dots: facile synthesis and application as a turn-off fluorescent probe for detection of $\mathrm{Hg}^{2+}$ ions. Biosensors and Bioelectronics, 55, 83-90

Zhang, Y., Chan, H. F., \& Leong, K. W. (2013). Advanced materials and processing for drug delivery: the past and the future. Advanced Drug Delivery Reviews, 65(1), 104-120. 